\title{
SUSTITUCIÓN DE MELAZA POR MUCÍLAGO DE CAFÉ (COFFEA ARABICA L.) EN BLOQUES NUTRICIONALES PARA RUMIANTES
}

\author{
REPLACEMENT OF MOLASSES BY COFFEE (COFFEA ARABICA L.) MUCILAGE \\ IN NUTRITIONAL BLOCKS FOR RUMINANTS
}

\author{
Pinto, R.R. ${ }^{{ }^{*}}$; Medina, J.A. ${ }^{1}$; Medina, F.J. ${ }^{1}$; Guevara, F. ${ }^{1}$; Gómez, H. ${ }^{1}$; Ley, A. ${ }^{1}$ y Carmona, J. ${ }^{2}$ \\ ${ }^{1}$ Facultad de Ciencias Agronómicas. Universidad Autónoma de Chiapas. Villaflores. Chiapas. México. \\ "pinto_ruiz@yahoo.com.mx \\ ${ }^{2}$ El Colegio de la Frontera Sur (ECOSUR). San Cristóbal de las Casas. Chiapas. México.
}

\section{PALABRAS CLAVE}

Suplementación estratégica. Recursos locales. Trópico.

\section{RESUMEN}

El objetivo de este trabajo fue evaluar la incorporación del mucílago, subproducto del café, en la fabricación de bloques nutricionales sobre sus variables de fabricación, composición química y preferencia animal. Se utilizaron bloques nutricionales elaborados con distintos porcentajes de inclusión de mucilago de café $(0,25,50,75$ y $100 \%$ ) en sustitución de la melaza, además de los ingredientes base como, maíz, cemento, urea, minerales y azufre. Se evaluaron las variables: días de fraguado, consistencia, facilidad de mezclado y dureza; la composición química se conoció a través del análisis de proteína cruda, insoluble y soluble, fibra detergente neutra, materia orgánica y cenizas. Para la prueba de preferencia animal se utilizó una prueba de cafetería, en la cual, a través de la observación continua, se realizaron las mediciones siguientes: número de visitas, número de lamidas o mordidas, tiempo efectivo de mordidas o lamidas y el consumo de cada bloque por cada animal. Con estas mediciones, se calcularon las siguientes variables: velocidad de lamida/mordida, velocidad de consumo, tamaño de lamida/mordida, tasa de consumo e índice de preferencia. Los datos de calidad y preferencia fueron sometidos a un análisis de varianza mediante el procedimiento GLM del paquete estadístico SAS (SAS, 1991). Para las variables de calidad se utilizó un diseño experimental completamente al azar con cuatro tratamientos y diez repeticiones por tratamiento. Para la prueba de preferencia, el experimento tuvo un período de

\section{AdDITIONAL KEYWORDS}

Strategic supplementation. Local resources. The tropics.

colecta de datos de cuatro días con dos periodos de evaluación. Se utilizó un diseño experimental cuadrado latino $4 \times 4$. En ambos casos, las medias fueron evaluadas utilizando la prueba de Tukey $(p<0,05)$. Los resultados indican que es factible la incorporación de mucilago de café en la fabricación y consumo de bloques nutricionales hasta en un $75 \%$ en sustitución de melaza de caña, ya que no se producen alteraciones en la composición química de éstos, además que la preferencia animal es mayor.

\section{SUMMARY}

The objective of this study was to evaluate the incorporation of the sub product mucilage of coffee in the fabrication of nutritional blocks and its impact on fabrication variables, chemical composition and animal preference. Nutritional blocks with different concentrations of coffee mucilage $(0$, 25, 50, 75 and $100 \%$ ) were used in place of molasses and combined with base ingredients corn, cement, urea, minerals and sulfur. The following variables were evaluated: setting time, consistency, ease of mixing, and hardness. The chemical composition was found by analyzing raw protein, insoluble and soluble protein, fractions of neutral detergent fiber, organic matter and ash. For the test of animal preference, a cafeteria test was used with the following measurements: number of visits, number of licks and bites, effective time of licking or biting, and consumption of each 
block by each animal. The field measurements were used to calculate the following variables: licking/biting speed, consumption speed, size of lick or bite, consumption rate and preference index. Data on quality and preference were submitted to an analysis of variance through the GLM procedure of the SAS statistical package (SAS, 1994). Quality variables were analyzed using a randomized experimental design with seven treatments and 10 repetitions per treatment. For the preference test, the experiment had a data collection period of four days, with two evaluation periods. A Latin square experimental design was used. In both cases, averages were evaluated using the Tukey test $(p<0.05)$. Results indicate that it is feasible to incorporate coffee mucilage in nutritional blocks as an up to $75 \%$ substitution for molasses from sugar cane. No alterations are thus produced in the chemical composition of the nutritional blocks and animal preference is greater than with molasses.

\section{INTRODUCCIÓN}

En Chiapas (zona tropical del sureste de México), la alimentación de los rumiantes se basa en el pastoreo de gramíneas nativas e introducidas, las cuales se ven afectadas tanto en su calidad nutricional como en su disponibilidad por las condiciones climáticas imperantes, sobre todo durante la época de estiaje, y es en estas condiciones cuando se recurren a una serie de estrategias de suplementación para mejorar la eficiencia de uso de los pastos fibrosos (Preston y Leng, 1987). Los bloques nutricionales, uno de los suplementos más utilizados en la región , presentan ventajas sobre la suplementación común. Para su formulación y fabricación se deben emplear ingredientes locales. Actualmente la melaza sirve de vehículo para la incorporación de la urea pero resulta costosa por unidad de nutriente y muchas veces es escasa, lo que limita su uso y, por tanto, son necesarias alternativas baratas que reemplacen a los ingredientes limitantes.

Al respecto, Chiapas cuenta con más de 245000 ha cultivadas de café, con más de 175000 familias, sobre todo indígenas (ztoztiles), involucradas en su cultivo, generando de 1,5 a 1,6 millones de sacos de café producidos anualmente, siendo líder mundial en la producción de café orgánico y un sólido participante en el comercio justo, ocupando el primer lugar de producción a nivel nacional (SAGARPA, 2011). Sin embargo, en la producción y procesamiento del café se generan grandes cantidades de subproductos (se estima que en el café cereza que se cosecha, el 39,4\% es pulpa, $21,6 \%$ es mucílago y el 10,4\% es cascarilla), que no se utilizan y son contaminantes del agua y suelo: aproximadamente, 82000 toneladas de mucílago de café, son vertidas anualmente a arroyos, ríos u otras fuentes de agua.

Se ha explorado con éxito la posibilidad de utilizar estos residuos en alimentación animal (rumiantes, cerdos y peces). La pulpa de café ha sido la mas frecuentemente incorporada a los sistemas de alimentación, principalmente de rumiantes, a través de su uso en silos; la cascarilla también ha sido empleada en dietas integrales (Noriega et al., 2008 y 2009) pero no existe información sobre el uso del mucílago (llamado algunas veces agua-miel). Por ello, es de interés estudiar su integración en los bloques nutricionales para la alimentación animal, ya que por su constitución liquida y su riqueza en azúcares reductores (glucosa) y no reductores (sacarosa) permite su incorporación en los bloques nutricionales y a la vez ser sustituto de la melaza, con la finalidad de reducir su impacto ambiental negativo, darle un valor agregado al subproducto y proporcionar al ganadero una alternativa de suplementación de menor costo. Por tanto, el objetivo de este trabajo fue evaluar la utilidad del mucilago de café en la fabricación de bloques nutricionales y su repercusión sobre la composición química y preferencia de éstos en los rumiantes.

\section{MATERIAL Y MÉTODOS}

La evaluación se realizó en la estación 
experimental de la Facultad de Ciencias Agronómicas de la Universidad Autónoma de Chiapas, en el municipio de Villaflores, perteneciente al estado de Chiapas, situado entre los $16^{\circ} 13^{\prime} 15^{\prime \prime} \mathrm{N}$ y $93^{\circ} 16^{\prime} 07^{\prime \prime} \mathrm{O}$, a una altitud de 610 m s.n.m. El clima es cálido subhúmedo, la temperatura media anual es de $24,3{ }^{\circ} \mathrm{C}$ y la precipitación media anual de $1,209 \mathrm{~mm}$, que ocurre principalmente en el verano (García, 1989).

El mucílago de café se obtuvo en comunidades indígenas cafetaleras aledañas al municipio de Jaltenango, localizado en la región Frailesca (Chiapas) en la cosecha de 2011 (diciembre), directamente del proceso de lavado del café y almacenado en recipientes de plástico para su uso inmediato.

Los bloques se realizaron según la metodología de Pinto y Ayala (2004), considerando los niveles de los ingredientes clave (melaza-urea), las características químicas de los mismos y el contenido de materia seca de la mezcla. Se utilizó la fórmula que mejor resultado ha proporcionado en la región (Pinto y Ayala, 2004) con grano de maíz (35,5 $\%)$, urea $(10 \%)$, cemento (16\%), minerales (10\%), azufre $(0,5 \%$, relación $1: 10$ con urea) y melaza (28\%). Los tratamientos evaluados se presentan en la tabla I, donde la melaza fue sustituida parcial o totalmente por el mucílago de café. Se fabricaron 10 bloques de $25 \mathrm{~kg}$ cada uno de ellos en cada tratamiento, los cuales fueron considerados como repeticiones.

Por observación directa, se evaluaron las siguientes variables de fabricación: facilidad de mezclado: esfuerzo en realizar la mezcla; dureza: la fuerza de penetración al suplemento; tiempo de fraguado: días en los que alcanzó la dureza adecuada y consistencia: distribución y agregación de los ingredientes en la mezcla, de acuerdo a la metodología empleada por Vázquez (2001). Cabe señalar, que el T5 no fue evaluado posteriormente, debido a que el bloque nutricional no logró la dureza adecuada.

La composición química, se analizó sobre 10 muestras compuestas (repeticiones), secadas en estufa de aire forzado a $60{ }^{\circ} \mathrm{C}$ y molidas individualmente en molino de motor tipo Wiley, obteniéndose submuestras de aproximadamente $300 \mathrm{~g}$ con un tamaño de molido aproximado de $2 \mathrm{~mm}$. Se determinaron proteína bruta (PB), materia orgánica (MO) y cenizas (CE) de acuerdo a los procedimientos de la AOAC (1990). La fracción de fibra detergente neutro (FDN) fue determinada mediante la técnica de Van Soest et al. (1991). Los valores de energía calórica (EC) se obtuvieron mediante bomba calorimétrica, basándose en combustión cerrada en presencia de oxígeno y lectura de incrementos de temperatura con termómetro calibrado (Analytical Methods for Oxygen Bombs, 1987). La proteína insoluble se obtuvo por el método Kjeldahl, mientras que la proteína soluble se obtuvo por diferencia entre proteína bruta y proteína insoluble (Sosa, 1979).

La preferencia animal se evaluó mediante una prueba de cafetería (Kaitho, 1997) utilizando para ello 4 corrales individuales, donde se confinaron 4 toretes Cebú-Suizo, encaste típico de la región, con un peso promedio de $235 \pm 12$,3 kgy una edad aproximada de dos años, con rumen totalmente desarrollado y sin antecedentes de consumo de bloques nutricionales. Previamente, cada animal fue desparasitado interna y externamente; además, se les aplicó un com-

Tabla I. Sustitución de la melaza por mucílago de café (Coffea arabica L.) en la fabricación de bloques nutricionales para rumiantes. (Substitution of molasses by coffee mucilage (Coffea arabica L.) in the fabrication of nutritional blocks for ruminants).

\begin{tabular}{ccc} 
Tratamientos & Melaza $\%$ & Mucilago de café $\%$ \\
\hline T1 & 100 & 0 \\
T2 & 75 & 25 \\
T3 & 50 & 50 \\
T4 & 25 & 75 \\
T5 & 0 & 100 \\
\hline
\end{tabular}


plejo vitamínico ADE. Los animales permanecieron en pastoreo de praderas de estrella africana (Cynodon plectostachyus) todo el día, por la tarde fueron confinados en los corrales (14:00 a18:00 h) con comederos de madera con cuatro compartimientos. A los animales confinados en su corral, les fueron ofrecidos los cuatro tratamientos de manera simultánea, colocados aleatoriamente en los compartimentos del comedero durante cada día de evaluación, de tal forma que ocuparan todos los lugares posibles dentro del comedero, con la finalidad de bloquear el hábito reflejo de los animales a la posición, la distancia del bloque y el primer encuentro con el suplemento. Los animales fueron adaptados a los bloques nutricionales durante un período de ocho días. A través de la observación continua, se realizaron las mediciones siguientes: número de visitas, número de lamidas o mordidas, tiempo efectivo de mordidas o lamidas y el consumo de cada bloque por el animal. Con las mediciones obtenidas en campo, se calcularon las siguientes variables: velocidad de lamida/ mordida, velocidad de consumo, tamaño de lamida/mordida, tasa de consumo e índice de preferencia siguiendo la metodología propuesta por Pinto et al. (2003).

Los datos de calidad y preferencia fueron sometidos a análisis de varianza mediante el procedimiento GLM del paquete estadístico SAS (SAS, 1994). Para analizar las variables de calidad se utilizó un diseño experimental completamente al azar con cuatro tratamientos (tipo de bloque) y 10 repeticiones por tratamiento (bloques). Para la prueba de preferencia, el experimento tuvo un período de colecta de datos de cuatro días con dos periodos de evaluación. Se utilizó un diseño experimental cuadrado latino 4x4. En ambos casos, las medias fueron evaluadas utilizando la prueba de Tukey $(\mathrm{p}<0,05)$.

\section{RESULTADOSYDISCUSIÓN}

En los bloques nutricionales para ru- miantes los componentes pueden sustituirse por otros que cumplan una función similar (Birbe et al., 1994). En la tabla II, se observa que, excepto en el tratamiento que incluyó $100 \%$ de mucílago, los tiempos de fraguado son los generalmente considerados aceptables (Echemendia, 1990). El menor tiempo de fraguado (24 h) se registró con 25 y $50 \%$ de inclusión de mucílago; el resto de los tratamientos (0 y $75 \%$ de inclusión) fraguó en 48 h. Pinto y Ayala (2004), refieren que las materias primas poseen compuestos que inciden sobre el efecto de fraguado del ligante, retardando o inhibiendo el fraguado, por ello el mucílago de café pudo influir en el tiempo de fraguado, aspecto posiblemente asociado a la densidad de la mezcla obtenida.

Sansoucy (1986) considera que la melaza debe ser de 85 o más grados brix para facilitar la solidificación de los bloques; en este caso, el mucilago de café que presenta 1,5 de grados brix, podría haber afectado a esta variable cuando es utilizado como único medio diluyente (T5).

La facilidad de mezclado, al elaborar los bloques puede estar relacionada con la

Tabla II. Variables de fabricación de bloques nutricionales formulados con diferentes pproporciones de mucílago de café(Coffea arabica L.). (Fabrication variables for nutritional blocks formulated with different levels of coffee mucilage (Coffea arabica L.)).

\begin{tabular}{crrrr} 
Tratamientos & TF, horas & C & FM & DU \\
T1 & 48 & S & B & D \\
T2 & 24 & S & B & D \\
T3 & 24 & S & B & D \\
T4 & 48 & V & MB & D \\
T5 & NF & V & MB & NE \\
\hline
\end{tabular}

TF= Tiempo de fraguado, horas; $\mathrm{C}=$ Consistencia; $\mathrm{FM}=$ Facilidad de mezclado; $\mathrm{DU}=$ Dureza.

$\mathrm{S}=$ Seca; $\mathrm{V}=$ Viscosa; $\mathrm{B}=$ Buena; $\mathrm{MB}=$ Muy buena; $D=$ Duro; $M D=$ Muy duro; NF= No fraguó; $N E=$ No endureció. 
Tabla III. Composición química de bloques nutricionales formulados con diferentes porcentajes de inclusión de mucilago de café (Coffea arabica L.). (Chemical composition of nutritional blocks formulated with different percentages of inclusion of coffee mucilage (Coffea arabica L.))

\begin{tabular}{|c|c|c|c|c|c|c|c|c|}
\hline & MS & PC & PS & PI & MO & CE & EC & FDN \\
\hline T1 & $86,9^{a} \pm 0,48$ & $25,6^{a} \pm 1,03$ & $23,1^{\mathrm{a}} \pm 1,74$ & $2,5^{a} \pm 0,78$ & $57,8^{a} \pm 0,19$ & $29,0^{c} \pm 0,34$ & $2,3^{\mathrm{ab}} \pm 0,06$ & $1,83^{\mathrm{ab}} \pm 0,11$ \\
\hline T2 & $87,0^{a} \pm 0,32$ & $25,7^{\mathrm{a}} \pm 0,24$ & $23,2^{a} \pm 0,93$ & $2,4^{a} \pm 0,83$ & $55,0^{\mathrm{b}} \pm 0,32$ & $32,0^{a} \pm 0,17$ & $2,3^{\mathrm{ab}} \pm 0,04$ & $1,5^{\mathrm{bc}} \pm 0,01$ \\
\hline T3 & $83,7^{\mathrm{bc}} \pm 0,87$ & $25,3^{a} \pm 0,17$ & $22,6^{a} \pm 1,68$ & $2,7^{a} \pm 1,52$ & $53,3^{\mathrm{c}} \pm 0,44$ & $30,4^{b} \pm 0,54$ & $2,3^{\mathrm{b}} \pm 0,02$ & $0,99^{c} \pm 0,00$ \\
\hline T4 & $80,5^{\mathrm{d}} \pm 0,45$ & $26,0^{\mathrm{a}} \pm 0,77$ & $24,2^{\mathrm{a}} \pm 0,10$ & $1,7^{a} \pm 0,71$ & $49,2^{\mathrm{c}} \pm 0,29$ & $31,3^{a b} \pm 0,53$ & $2,2^{\mathrm{b}} \pm 0,06$ & $1,83^{\mathrm{ab}} \pm 0,43$ \\
\hline
\end{tabular}

Medias en la misma columna con letras distintas difieren estadísticamente (Tukey $p<0,05)$.

MS= Materia seca; $\mathrm{PC}=$ Proteína cruda; $\mathrm{PS}=$ Proteína soluble; $\mathrm{PI}=$ Proteína insoluble; $\mathrm{MO}=$ Materia orgánica; $\mathrm{CE}=$ Cenizas; $\mathrm{EC}=$ Energía calorífica; FDN= Fibra detergente neutra.

consistencia: cuando era seca, la facilidad de mezclado fue buena, pero en los mas altos niveles de mucílago mejoró, así como la consistencia del bloque (viscoso); sin embargo, los bloques con este nivel de inclusión de mucílago no lograron fraguar después de veinte días de la elaboración. Todos los tratamientos (excepto T5) que se presentan en la tabla II tuvieron un aspecto sólido, lo que indica que los diferentes niveles de mucilago no afectaron esta variable. Lograr la dureza en los bloques nutricionales en un periodo de 24 o 48 horas, es una ventaja que permite utilizarlos en un tiempo corto después de la elaboración y además facilita su manejo sin riesgo de deformaciones o rupturas (Pinto y Ayala, 2004).

La composición química se describe en la tabla III. La proteína, el componente más importantes en este tipo de suplemento (Leng y Preston, 1983), fue similar en los diferentes tratamientos ( $p>0,05)$, con un promedio de 25,49\%; tambien fueron similares los niveles de proteínas soluble (PS) e insoluble (PI), cuyas medias fueron de 22,91 y $2,55 \%$, respectivamente. Las características nutricionales de la melaza le confieren una alta solubilidad en el rumen, lo que coincide de forma favorable con la alta solubilidad de la urea, por lo que el mucílago de café podría ser considerado un vehículo apropiado para ésta.
De acuerdo con la escasez de MO en el mucilago, los valores de MO y EC disminuyen $(\mathrm{p}<0,05)$ al aumentar la proporcion de mucílago.

En la tabla IV se aprecian diferencias $(\mathrm{p}<0,05)$ para la velocidad de lamidas (VL) y velocidad de consumo (VC), pero no para el

Tabla IV. Conducta ingestiva de bovinos suplementados con bloques nutricionales con distintas proporciones de mucílago de café (Coffea arabica L.). (Eating behavior of bovines supplemented with nutritional blocks with different levels of coffee mucilage (Coffea arabica L.)).

\begin{tabular}{ccccc}
\hline Tratamientos & VL & VC & TL & TC \\
\hline T1 & $1,91^{\mathrm{b}}$ & $0,14^{\mathrm{bc}}$ & $0,31^{\mathrm{a}}$ & $30,96^{\mathrm{a}}$ \\
T2 & $0,65^{\mathrm{b}}$ & $0,08^{\mathrm{c}}$ & $0,12^{\mathrm{a}}$ & $12,29^{\mathrm{a}}$ \\
T3 & $0,97^{\mathrm{b}}$ & $0,41^{\mathrm{b}}$ & $0,38^{\mathrm{a}}$ & $48,02^{\mathrm{a}}$ \\
T4 & $30,62^{\mathrm{a}}$ & $6,31^{\mathrm{a}}$ & $0,21^{\mathrm{a}}$ & $22,74^{\mathrm{a}}$ \\
& & & & \\
Media & 8,54 & 1,74 & 0,25 & 28,50 \\
EEM & 3,72 & 0,77 & 0,05 & 6,14 \\
\hline
\end{tabular}

Medias en la misma columna con letras distintas difieren estadísticamente (Tukey, $\mathrm{p}<0,05$ ).

$\mathrm{VL}=$ Velocidad de lamida (lamidas por minuto); VC= Velocidad de consumo (g MS por minuto); TL= Tamaño de lamida (g MS por lamida); TC= Tasa de consumo (g MS minunto efectivo de consumo); $\mathrm{EEM}=$ Error estándar de la media. 
Tabla $\boldsymbol{V}$. Índice de preferencia de bovinos consumiendo bloques nutricionales distintas proporciones de mucílago de café(Coffea arabica L.). (Preference index of bovines consuming nutritional blocks with different levels of coffee mucilage (Coffea arabica L.)).

\begin{tabular}{lcccc}
\hline Tratamientos & T1 & T2 & T3 & T4 \\
\hline $\begin{array}{lcccc}\text { consumo } \\
\text { visitas }\end{array}$ & $0,126^{\mathrm{b}}$ & $0,117^{\mathrm{b}}$ & $0,144^{\mathrm{b}}$ & $0,613^{\mathrm{a}}$ \\
& $0,018^{\mathrm{b}}$ & $0,0135^{\mathrm{b}}$ & $0,0603^{\mathrm{b}}$ & $0,91^{\mathrm{a}}$
\end{tabular}

Medias en la misma hilera con letras distintas difieren estadísticamente (Tukey, $\mathrm{p}<0,05$ ).

tamaño de lamida (TL) y tasa de consumo (TC).

Si se considera la TC (cantidad de bloque consumido por unidad de tiempo efectivo de consumo) como la variable más importante (Alonso et al., 2009), los resultados presentados en la tabla IV indican que es factible incluir el mucilago de café en la formulación del bloque hasta en un $75 \%$, ya que los valores obtenidos para esta variable, y además los valores de TL, fueron similares entre tratamientos $(\mathrm{p}>0,05)$. Por otro lado, la velocidad con la que el bloque fue consumido (VL y VC) explica diferencias en la conducta animal sobre el uso de este tipo de suplemento, es decir, los animales consumen de manera más rápida los bloques fabricados con $75 \%$ de mucilago, resultado posiblemente asociado al sabor del conferido por el mucílago, aspecto que toma interés en los procesos de adaptación al suplemento por parte del animal; sin embargo, la cantidad de bloque ingerido por lamida, así como la cantidad ingerida por

\section{BIBLIOGRAFÍA}

Alonso D.M.; Torres, A.J.F.; Sandoval, C.A.; Hoste, H.; Aguilar, C.H. and Capetillo, L. C. 2009. Sheep preference for different tanniniferous tree fodders and its relationship with in vitro gas production and digestibility. Anim Feed Sci Technol, 151: 75-85. tiempo efectivo fueron similares en todos los tratamientos. Las distintas características físico-químicas de los suplementos hace que las velocidades de consumo sean diferentes (Salas et al., 2001), al respecto, se encontró una relación negativa entre la VC y el contenido de $P I\left(R^{2}=-0,96 ; p<0,05\right)$. Por otro lado, en la tabla $\mathbf{V}$, se observa que el tratamiento más preferido por el animal fue el de $75 \%$ de inclusión de mucilago $(\mathrm{p}<0,05)$, respuesta asociada a varios factores, tales como el sabor, olor, textura, composición química, pero el animal es el mejor indicador (Kaitho, 1997). Estos resultados corroboran lo encontrado en la prueba de conducta ingestiva realizada, además se demuestra la importancia de involucrar el componente animal en las evaluaciones iniciales de cualquier alimento.

\section{CONCLUSIONES}

Es factible la incorporación de mucilago de café en la fabricación de bloques nutricionales hasta un $75 \%$ en sustitución de melaza de caña, ya que no se alteran las variables de fabricación ni se producen alteraciones en su composición química, además de que la preferencia animal es mayor.

\section{AGRADECIMIENTOS}

Los autores agradecen al Sistema Institucional de Investigación de la Universidad Autónoma de Chiapas en su $10^{\text {a }}$ convocatoria (SINNV-UNACH 2011) por el financiamiento otorgado al proyecto titulado 'Usos de los residuos derivados de la producción del café Coffea arabica (Pulpa, mucílago y cascarilla)'.

Analytical Methods for Oxygen Bombs. 1987. PARR Instrument Company. Illinois, EUA. Serie No. 207M. 8 pp.

AOAC. 1990. Official Methods of Analysis of the Association of Official Analytical Chemists. $15^{\text {th }}$ ed. Washington, DC. USA. 500 pp. 


\section{USO DE MUCILAGO DE CAFÉ EN LOS BLOQUES NUTRICIONALES}

Birbe, B.; Chacón, E.; Taylhardat, L.; Garmendia, J. y Mata, D. 1994. Aspectos físicos de importancia en la fabricación y utilización de bloques multinutricionales. En: A. Cardozo y B. Birbe (Eds.). Bloques multinutricionales. I. Conferencia Internacional. Guanare. Venezuela. pp. 1-14.

Echemendia, M. 1990. Metodología para la elaboración de bloques multinutricionales. Tesis de especialidad en alimentación y manejo de ganado bovino. Instituto de Ciencia Animal. La Habana. Cuba. 49 pp.

García, E. 1989. Modificación al sistema de clasificación climática de Köppen. Universidad Nacional Autónoma de México. México. DF. 246 pp.

Kaitho, R.J. 1997. Nutritive value of browses as protein supplement(s) to poor quality roughages. Ph.D. Tesis. Department of Animal Nutrition. Wageningen Agricultural University. Wageningen. The Netherlands. 190 pp.

Leng, R.A. and Preston, T.R. 1983. Nutritional trategies for the utilization of agroindustrial byproducts by ruminants and extension of the principles and technologies to the small farmers in Asia. Proceedings of the Fifth World Conference on Animal Production. 1: 310-318.

Noriega, S.A.; Silva, A.R. y García, M. 2008. Utilización de la pulpa de café en la alimentación animal. Zootec Trop, 26: 411-419.

Noriega, S.A.; Silva, A.R. y García, M. 2009. Composición química de la pulpa de café a diferentes tiempos de ensilaje para su uso potencial en la alimentación animal. Zootec Trop, 27: 135-141.

Pinto, R.R.; Gómez, H.; Hernández, A.; Medina, F.;
Aguilar, V.H.; Nahed, J. y Carmona, J. 2003. Preferencia ovina de árboles forrajeros del centro de Chiapas, México. Past Forr, 26:181190.

Pinto, R.R. y Ayala, A. 2004. Los bloques nutricionales en la ganadería tropical. $1^{\mathrm{a}} \mathrm{ed}$. Edit. Fundación PRODUCE CHIAPAS. Universidad Autónoma de Chiapas. México. 95 pp.

Preston, T.R. and Leng, R.A. 1987. Matching ruminant production systems with available resources in the tropics and sub-tropics. Penambul Books. Armidale, Australia. pp. 193-196.

Sansoucy, R. 1986. Fabricación de bloques melaza-urea. Rev Mund Zootec, 57: 40-48.

SAS. 1994. User's guide. $4^{\text {th }}$ ed. Statistical Analysis Institute. Inc. North Carolina. USA. 470 pp.

SAGARPA. 2011. Producción de café cereza en Chiapas. Servicio de Información Agroalimentaria y Pesquera. México. 123 pp.

Salas, A.; San Martín, F. y Carcelen F. 2001. Preferencia y consumo en ovinos y su relación con las características físicas de los bloques nutricionales. Rev Inv Vet Perú, 12: 87-98.

Sosa, E. 1979. Manual de procedimientos analíticos para alimentos de consumo animal. Departamento de Zootecnia. Chapingo. México. 76 pp.

Vázquez, P.M. 2001. Elaboración y calidad de bloques nutricionales para rumiantes utilizando ingredientes regionales. Tesis de Licenciatura. Universidad Autónoma de Chiapas. México. $115 \mathrm{pp}$.

Van Soest P.J.; Robertson, J.B and Lewis, B.A. 1991. Methods for dietary fiber, neutral detergent fiber and non-starch polysaccharides in relation to animal nutrition. J. Dairy Sci, 74: 3583-3597. 